\title{
ERRATUM
}

\section{Competence-based Vocational and Professional Education}

\section{Bridging the Worlds of Work and Education}

\author{
Martin Mulder \\ (C) Springer International Publishing Switzerland 2017 \\ M. Mulder (ed.), Competence-based Vocational and Professional Education, \\ Technical and Vocational Education and Training: Issues, Concerns and \\ Prospects 23, DOI 10.1007/978-3-319-41713-4
}

DOI 10.1007/978-3-319-41713-4_51

\section{Erratum to:}

Chapter 37 in: Michael Sailer, Jan Hense, Heinz Mandl, and Markus Klevers, Fostering Development of Work Competencies and Motivation via Gamification, DOI 10.1007/978-3-319-41713-4_37.

The original version of this chapter was inadvertently published with incorrect $F$ values in the results of MANCOVA listed in page 811 and 812. The comma in the brackets was erased which resulted " $F(1100)$ " instead of " $F(1,100)$ " 
The value of 21.98 was published incorrectly as 21,98 in the original version of the book. It is updated in page 811 .

- Regarding quantitative performance, the gamification group achieved 62.44 $(S D=15.92)$ picks on average, while the control group achieved 46.82 $(S D=18.92)$ picks. A MANCOVA indicates that this quantitative performance is significantly higher in the gamification group than in the control group, $F(1,100)=72.49, p<.01, \eta_{\mathrm{p}}^{2}=.420$.

- Regarding qualitative performance, the gamification group achieved an accuracy rate of $94 \%(S D=.07)$ on average. The control group achieved an accuracy rate of $87 \%(S D=.14)$ on average. A MANCOVA indicates that this qualitative performance is significantly higher in the gamification group than in the control group, $F(1,100)=21.98, p<.01, \eta_{\mathrm{p}}{ }^{2}=.180$.

2. To what extent can gamification work-integrated training environments foster experiences of competence, autonomy regarding task meaningfulness, autonomy regarding decision freedom and relatedness?

- Regarding the experience of competence, the gamification group scored 4.81 $(S D=1.40)$ on a seven-point Likert scale, while the control group scored 4.11 $(S D=1.13)$. A MANCOVA indicates that participants from the gamification group have significantly higher experiences of competence than participants from the control group, $F(1,100)=8.11, p<.01, \eta_{\mathrm{p}}{ }^{2}=.075$.

- Regarding the experience of task meaningfulness (autonomy), the gamification group scored $5.46(\mathrm{SD}=1.06)$ on a seven-point Likert scale, while the control group scored $4.34(S D=1.38)$. A MANCOVA indicates that participants from the gamification group have significantly higher experiences of task meaningfulness than participants from the control group, $F(1,100)=18.90$, $p<.01, \eta_{\mathrm{p}}^{2}=.159$.

- Regarding the experience of decision freedom (autonomy), the gamification group scored $4.03(\mathrm{SD}=1.49)$ on a seven-point Likert scale, while the control group scored $3.64(S D=1.58)$. A MANCOVA indicates that participants from the gamification group have significantly higher experiences of decision freedom than participants from the control group, $F(1,100)=4.03, p<.05$, $\eta_{\mathrm{p}}^{2}=.039$.

- Regarding the experience of relatedness, the gamification group scored 3.31 $(S D=1.47)$ on a seven-point Likert scale, while the control group scored 1.93 $(S D=.99)$. A MANCOVA indicates that participants from the gamification group have significantly higher experiences of relatedness than participants from the control group $F(1,100)=27.85, p<.01, \eta_{\mathrm{p}}{ }^{2}=.218$. 


\section{Erratum to:}

Chapter 48 in: Susan Seeber and Eveline Wittmann, Social Competence Research: A Review, DOI 10.1007/978-3-319-41713-4_48.

The original version of this chapter was inadvertently published without reference for figure 1. The reference is updated in the reference list as : "Kanning, U. (2009b). Inventar sozialer Kompetenzen. Göttingen: Hogrefe". 